\title{
ANÁLISE DOS MODOS DE FALHA E SEUS EFEITOS NO PROCESSO DE PRODUÇÃO DE MANCAIS
}

\section{FAILURE MODES AND EFFECTS ANALYSIS ON THE PRODUCTION PROCESS OF BEARINGS}

\author{
André Victor Traleski ${ }^{1}$; Henry Stepien ${ }^{2}$; Ricardo Augusto Blauth ${ }^{3}$ \\ ${ }^{1}$ Faculdade União - Ponta Grossa - Paraná - Brasil \\ andre_traleski@hotmail.com \\ ${ }^{2}$ Faculdade União - Ponta Grossa - Paraná - Brasil \\ henryst@bol.com.br \\ ${ }^{3}$ Universidade Positivo - Curitiba - Paraná - Brasil \\ ricardo@eqpconsultoria.com.br
}

\begin{abstract}
Resumo
A crescente competitividade em todos os setores da economia e a necessidade de aprimoramento da produção evitando desperdícios, aumentando a qualidade e produzindo a um preço atrativo, tem ganhado espaço nas discussões de empresas, de todos os segmentos, para buscar técnicas e metodologias que auxiliem alcançar estes objetivos. Uma das metodologias largamente empregada para esta gestão de riscos é a FMEA (Failure Mode and Effect Analysis - Análise dos Modos e Efeitos de Falhas), que visa identificar pontos possíveis de falha de um projeto, processo, produto ou serviço. Este trabalho foi elaborado em decorrência de um problema que afeta indústrias de vários setores, incluindo de metalurgia: o retrabalho no processo produtivo. O retrabalho implica na repetição de uma atividade causada por problemas de falha de mão de obra, material, problemas no processo ou projeto. No processo de fabricação de mancais, há uma enorme gama de variáveis que podem influenciar o resultado final e gerar um impacto significativo na qualidade do produto. Para suprimir esse impacto e adquirir uma vantagem competitiva no mercado foi selecionada a FMEA, como método para reduzir falhas no processo. O objetivo deste artigo é elaborar um planejamento de acordo com a metodologia da FMEA e propor planos de ações para os problemas identificados no processo de produção. Tem também como finalidade, demonstrar a eficácia da metodologia em disseminar uma cultura voltada para a melhoria contínua.
\end{abstract}

Palavras-chave: análise; falha; riscos; mancais; fluxograma.

\section{Introdução}

A crescente competitividade entre os mercados, principalmente entre as economias emergentes, traz uma grande preocupação especialmente para as indústrias brasileiras. Grandes produtores como a China, estão cada vez mais ganhando espaço no mercado nacional com produtos a preços mais competitivos que os produtos fabricados internamente. Com esta grande concorrência e visão holística para melhorias de produtos, a metodologia de Análise dos Modos de Falhas e seus 
Efeitos (FMEA) é uma das grandes técnicas utilizadas pelas empresas para detectarem falhas em seus projetos, processos, produtos e serviços e assim, manterem-se competitivas, reduzindo problemas, desperdícios e aumentando o lucro.

A FMEA foi desenvolvida na década de 60 para avaliação de projetos identificando possíveis modos de falhas na indústria aeroespacial e na década de 70 passou a fazer parte das investigações de falhas das indústrias aeronáuticas e nucleares. Com grandes resultados nestas indústrias, o setor automotivo também adotou esta metodologia que se estendeu para os fornecedores de peças automotivas, passando a ser uma exigência para certificação da ISO/TS 16949, uma das principais normas do segmento (AIAG, 2008, p. 1; ABNT, 2004, p. 1; VASCONCELLOS JR; MIGUEL, 1999, p. 2).

A metodologia visa identificar as falhas ou possíveis modos de falha em diferentes etapas de concepção de um produto ou serviço, relacionando os efeitos que podem ocasionar para os clientes e as possíveis causas destas falhas (MARSHALL, 2010, p. 169-170). Deste modo, entendendo quais são os pontos críticos pode-se estabelecer um plano de ação de melhorias com intuito de mitigar problemas e aplicar maiores controles evitando assim, a insatisfação do cliente ou desperdícios em qualquer etapa. É uma técnica que apresenta risco reduzido e eficiência na identificação, prevenção e resolução dos problemas, devido a característica de buscar identificar as causas raízes de cada falha e classificar quanto aos graus de ocorrência, severidade e detecção, gerando um índice de priorização de risco (GPR). (VASCONCELLOS JR; MIGUEL, 1999, p. 2; VINODH; SANTHOSH, 2012, p. 260).

Com a visão holística, as indústrias fornecedoras da cadeia automotiva e também de outros segmentos optaram por implementar a FMEA como uma ferramenta de gestão de riscos. Dentre estas, estão às fabricantes de bronzinas e mancais hidrodinâmicos, que produzem estes materiais de suporte e deslizamento de eixos para os segmentos automotivos, petróleo e gás, eólico, usinas sucroalcooleiras, metal-mecânica, entre outros setores. Os mancais são utilizados por estas diferentes indústrias, como elemento de apoio de eixos rotativos, deslizantes ou oscilantes e atuam também com a função de reduzir o atrito destas peças do conjunto mecânico.

O objetivo geral deste estudo é a identificação dos modos de falhas no processo de produção de mancais especiais, com a implementação da FMEA piloto no setor de fundição de uma metalúrgica situada na cidade de Ponta Grossa - Paraná e a difusão da metodologia para o grupo multifuncional formado.

De forma complementar, os objetivos específicos são: disseminar a cultura de analisar as falhas e seus efeitos em todo o ambiente fabril da metalúrgica, elaborar indicadores complementares para controle do processo e sugerir ações de melhoria a partir do estudo desenvolvido. 


\section{Fundamentação teórica}

\subsection{Gestão de risco e FMEA}

A indústria nacional atualmente vem ocupando um lugar de destaque no cenário econômico internacional, no qual, grandes oportunidades surgem a cada dia para empresas que conseguem criar um diferencial competitivo e estabelecer processos de produção mais eficientes. Ao mesmo tempo em que enfrentamos uma série de desafios originários da concorrência estrangeira, torna-se imprescindível a utilização de técnicas de melhoria da qualidade que ajudam a estabelecer um planejamento mais eficiente, utilizando melhor os recursos e aprimorando a comunicação interorganizacional.

Para Toledo et al (2013, p. 159), a melhoria da qualidade deve estar na rotina e inerente a todos os processos de uma organização. Todas as atividades que agreguem ou não valor, ou seja, produtivas ou administrativas, podem e devem ser continuamente avaliadas e melhoradas.

Para conseguir resultados eficazes e mensuráveis é necessário fazer uso de metodologias que busquem eliminar defeitos, aumentem a satisfação dos clientes e proponham ações de melhoria contínua. A qualidade passou a fazer parte do contexto das empresas e tem se demonstrado ser o meio mais condizente para a sobrevivência das empresas a médio e longo prazo. Uma maneira de uma empresa diferenciar-se é prestando serviços de alta qualidade e fornecendo produtos que ultrapassem as expectativas do cliente. De acordo com Hargreaves et al (2001, p.12), "a qualidade de um produto ou de um serviço é medida pelo conjunto de características capazes de atender as necessidades implícitas e explícitas do cliente".

Dentro desse contexto de globalização, dinamismo do mercado e controle de processos, surge uma questão determinante para o resultado das organizações: como lidar com as incertezas e riscos presentes no mercado e quais serão seus impactos na empresa? Uma prática amplamente conhecida no mercado financeiro, o gerenciamento de riscos, vem se destacando em outros segmentos e ajudando os administradores na tomada de decisões referentes ao cumprimento de leis e regulamentos, atingimento de metas e avaliação de riscos.

Segundo Brasiliano (2009, p.11-12), o gerenciamento de riscos corporativos deve gerar valor às partes interessadas, tendo isso como premissa desta gestão. Todas as organizações enfrentam incertezas, e os seus administradores devem ser críticos e bem embasados em suas decisões para determinar até que ponto aceitar essa incerteza, assim como prever as consequências para a geraração de valor às partes interessadas.

Para auxiliar as organizações na gestão de riscos, foi criada a ISO 31000:2009, que estabelece princípios, estruturas e um processo para gerir qualquer tipo de risco. De acordo com a NBR ISO 31000:2009 (2009, p.5), as organizações de todos os tipos e tamanhos sofrem influências 
que não garantem se será possível atingir estes objetivos e o tempo despendido para que isso ocorra. O efeito da incerteza proporciona uma condição conhecida como "risco" e que deve ser gerenciada pelas organizações para sobrevivência da mesma.

Um diferencial para as empresas é a realização de um bom planejamento durante a fase de desenvolvimento do projeto, que evite que ocorram falhas potenciais, ou seja, que detecte falhas antes e durante o funcionamento do projeto, isentando o cliente de possíveis transtornos.

A FMEA é uma ferramenta que utiliza variáveis qualitativas para realizar uma análise de possíveis modos de falha que podem originar-se em componentes e gerar efeito sobre a função de todo o conjunto, ou seja, permite minimizar ou erradicar as falhas, evitando assim, os seus efeitos.

De acordo com Helman e Andery (1995, p.25), a FMEA é um método de análise de projetos, sendo estes de produtos, processos, administrativos ou serviços, usado para identificar todos os possíveis modos potenciais de falha e determinar o efeito de cada um sobre o desempenho do sistema. Atualmente, pode ser considerada uma das melhores análises de confiabilidade no estágio de projeto para sistemas de engenharia de geração de energia (ARABIAN-HOSEYNABADI; ORAEE; TAVNER, 2010, p.817).

A FMEA (produto/processo) deve ser implementada a partir da formação de uma equipe multifuncional que irá definir as características do produto ou processo e irá relacionar as falhas que poderão ocorrer, descrevendo para cada tipo de falha, seus efeitos. A participação de especialistas de diferentes áreas amplia o número de informações e considerações, podendo obter uma avaliação mais aprofundada, com maiores condições de sucesso (ROTONDARO et al, 2002, p.157).

Para iniciar a elaboração de uma FMEA de processo, primeiro é necessário entender como este processo funciona e como as operações estão relacionadas, podendo isto ser feito por um mapeamento de processo resultando em um fluxograma das operações. Após definir cada uma das funções das operações, é preciso realizar um estudo dos requisitos que aquele determinado processo dever atender ou não para assegurar as qualidades do produto (VINODH; SANTHOSH, 2012, p. 261). Após entender o conceito e importância de cada operação, pode-se iniciar com a equipe um levantamento de modos de falhas para cada etapa do processo produtivo. É importante entender que o modo de falha, não é a causa, mas sim como esta falha ocorre (ARABIAN-HOSEYNABADI; ORAEE; TAVNER, 2010, p.818). Para a FMEA de processo, frequentemente um efeito pode estar ligado à causa raiz de uma falha em uma operação subsequente (VINODH; SANTHOSH, 2012, p. 267).

$\mathrm{Na}$ etapa de integração entre modo de falha, efeito e causa, o raciocínio e visão abrangente da equipe devem estar aguçados para conseguirem identificar todas as possibilidades e analisar criticamente os resultados de dados já mensurados se o processo já estiver ocorrendo. 
Posteriormente, é preciso atribuir índices de severidade, ocorrência e detecção para avaliar os riscos e estabelecer um método de priorização dos modos de falhas em função de seus efeitos e dos controles existentes para evitar que o problema chegue ao cliente, preparando então o plano de ação e discutindo melhorias (ROTONDARO, et al, 2002, p.157-160).

Este critério de atribuição de valores aos índices de severidade, detecção e ocorrência, pode ser variável, mas uma escala muito utilizada e de mais fácil aplicação é a atribuição de valores de 1 a 10, como exemplificado na tabela 1 seguinte:

Tabela 1: Exemplos de critérios para determinação de índices de ocorrência, severidade e detecção.

\begin{tabular}{ccc}
\hline Probabilidade de Ocorrência & Severidade & Probabilidade de Detecção \\
\hline Muito remota -1 & & Muito Alta -1 \\
Muito pequena -2 & Apenas perceptível -1 & Alta $-2,3$ \\
Pequena -3 & Pouca Importância $-2,3$ & Moderada $-4,5,6$ \\
Moderada $-4,5,6$ & Moderadamente Grave $-4,5,6$ & Pequena $-7,8$ \\
Alta -7.8 & Grave $-7,8$ & Muito Pequena -9 \\
Muito Alta $-9,10$ & Extremamente Grave $-9,10$ & Remota - 10 \\
\hline
\end{tabular}

Fonte: Rotondaro et al (2002, p.159).

O critério de priorização de falhas origina-se do grau de priorização de riscos (GPR) que é obtido pela multiplicação dos valores de cada índice, ou seja, quanto maior forem os valores de cada um dos índices, mais crítico será o GPR (AIAG, 2008, p.10-18; ROTONDARO et al, 2002, p.157-160; VINODH; SANTHOSH, 2012, p.260). A partir do critério de priorização de falhas pode se elencar as operações com maior criticidade, das quais se deve iniciar um plano de ação de melhorias. Porém considerar apenas o GPR pode não ser sempre a melhor opção, porque algumas etapas de alto grau de severidade, podem também afetar fortemente o produto final, assim como uma recorrência muito grande podem aumentar insatisfação do cliente se os defeitos chegarem até ele com frequência continua. Portanto cada modo de falha deve também ser avaliado individualmente. (VINODH; SANTHOSH, 2012, p. 267).

Seguindo a metodologia, o próximo passo é identificar os métodos presentes no processo para detectar as falhas e quais são os controles já empregados para atenuar estes problemas. O uso de indicadores de conformidade de produto ou de processo pode ser uma técnica útil de análise para avaliar se estes controles estão sendo eficientes e efetivos.

Para implementação da FMEA, a utilização do ciclo PDCA é bastante útil por estabelecer uma sequência lógica de ações e de como este projeto deve ser conduzindo para se obter melhores resultados. O ciclo PDCA, criado por Walter A. Shewhart e difundido por Willian E. Deming, é um método interativo de gestão para o controle das atividades de uma organização voltadas para a melhoria contínua. Partindo deste princípio, a etapa de Planejar (Plan) será conduzida pela identificação do problema e quantificação de desempenho do processo, decompondo as operações em funções e pontuando quais são os requisitos para cada etapa de pesquisa e análise dos modos de falha, suas causas e efeitos. Tendo finalizado este procedimento, ainda no Planejar, procede-se a 
interpretação e priorização das falhas e elaboração de um plano de ação para propor melhorias. Na sequência do Fazer (Do), os envolvidos na aplicação do plano de ação devem executar conforme as ações planejadas e serem treinados, não se esquecendo de realizar uma coleta de dados para avaliar as melhorias. Para Verificação (Check), os dados coletados devem ser comparados com a meta e se os resultados não alcançarem os objetivos projetados, o ciclo deve ser novamente executado, caso contrário, procede-se a elaboração da documentação e padronização da metodologia, mantendo sempre um estado de melhoria contínua e revisão da FMEA (BLAUTH; BLAUTH, 2009, p.316317; WERKEMA, 1995, p. 287-295).

A FMEA traz a organização um melhor conhecimento dos problemas e auxilia no estabelecimento de um sistema de prioridades de melhorias, investimento, desenvolvimento, análise-teste e validação. Com a aplicação desta ferramenta forma-se um arquivo de referência para futuros projetos, treinamentos, além do incentivo para a necessidade constante de desenvolvimento.

Rotondaro (2002, p.54-62) evidencia que esta ferramenta pode ser utilizada novamente com o intuito de se estabelecer um ciclo de melhoria contínua na empresa. Logo, torna-se um método importante para a garantia da qualidade. Outro benefício da FMEA é estruturar a empresa no sentido de criar uma atitude de prevenção de falhas, cooperação e trabalho em equipe e monitoramento do processo dentro da organização.

Essa metodologia faz com que a empresa mantenha sempre o foco no cliente, garantindo sua satisfação e segurança. A voz do cliente deve ser analisada para ter acesso à informação sobre o que é prioritário, para facilitar a identificação das características críticas para a qualidade. A capacidade de entender o que os clientes desejam e adquirir conhecimentos com ele é com certeza o caminho para estar à frente da concorrência, além de estabelecer uma linha de comunicação eficiente entre ambos. Tratar clientes de forma diferente, conquistar sua confiança, perceber suas necessidades e agir de acordo com isso é um modo de agregar mais valor para a empresa também. Empresas inovadoras já compreenderam a importância desse fato e desenvolvem estratégias para melhorar sua eficiência.

A imagem é o principal ativo da empresa e é através dela que são enviadas informações para os clientes, as quais irão orientá-los na tomada de decisão de compra ou contratação de um bem ou serviço.

\subsection{Ferramentas complementares}

Ao implementar a FMEA, é necessário utilizar simultaneamente outras ferramentas como por exemplo o diagrama de causa e efeito, gráfico de Pareto, Brainstorming, entre outros que facilitem e auxiliem na interpretação de dados. Para identificar e analisar as causas dos problemas o 
uso de ferramentas estatísticas permite priorizar os problemas por grau de importância, produzindo assim, melhores resultados.

Para Toledo et al (2013, p.206), o gráfico de Pareto representa uma série de dados sobre determinado problema e que possibilita identificar quais são os aspectos de maior influência a serem analisados e trabalhados. É um método com um retorno visual de quais são os fatores de maior importância, através do ordenamento dos índices de problema em uma escala crescente ou decrescente, sejam eles tipos de defeitos, tipos de produtos ou mesmo projetos mais problemáticos.

Aliado a FMEA e ao diagrama de causa e efeito está o Brainstorming ou "tempestade de ideias". Esta é uma técnica usada para dar suporte a outras ferramentas de gestão e que estimula a criatividade de um indivíduo ou grupo para problemas que tem muitas soluções possíveis, avaliando e selecionando as melhores ideias com a finalidade de chegar a um denominador comum. Segundo Toledo et al (2013, p.208), essa técnica tem uma característica de potencializar a criatividade de todas as pessoas que participam para que se expressem, de modo voluntário e sem críticas externas ou dos participantes do grupo, todas as ideias que possam ter relação com o assunto abordado e que posteriormente serão filtradas.

Para entender a relação entre um resultado de um processo (efeito) e os fatores (causas), o diagrama de Ishikawa ou diagrama de causa e efeito é uma ferramenta muito utilizada. Este diagrama, elaborado em formato de uma espinha de peixe, classifica as causas de um problema em grupos sendo estes conhecidos como 6M (mão de obra, meio ambiente, método, material, máquina, medida). A partir do Brainstorming, possíveis causas geradoras daquele efeito, são elencadas em cada um dos ramos do 6M, facilitando avaliar de onde surge o problema (BLAUTH; BLAUTH, 2009, p.147-154; WERKEMA, 1995, p.101-115).

Seguindo o ciclo do PDCA, após identificar os problemas, as possíveis causas e os efeitos, é preciso elaborar um plano de ação para contenção dos problemas e melhoria do processo. Para concretizar um projeto é preciso um plano de execução de tarefas, que coloque em prática tudo o que foi desenvolvido anteriormente. Através do 5W2H é fornecida uma visão do que será feito, por quem, quando, onde e por que será feito, além dos custos que serão geridos durante o processo. É um método de descrever as ações atribuindo responsabilidades e prazos (WERKEMA, 2002, p.207).

\subsection{Processo de produção de mancais}

Os mancais são elementos de máquina utilizados para reduzir o atrito e o desgaste superficial entre peças rígidas e trabalhar como apoio de um eixo promovendo o movimento suave entre superfícies sólidas (ARNOLD, 2005, p. 7-8). Estes mancais são utilizados em máquinas rotativas como compressores, turbinas, bombas, geradores elétricos e motores elétricos. Uma 
diversidade de segmentos utilizam estes elementos de máquina, como indústrias automobilísticas, de petróleo e gás, químicas, alimentícias, produtoras de metais e de cerâmicas, usinas hidrelétricas e eólicas, usinas sucroalcooleiras, entre outras. Estes mancais podem ser produzidos por processos de laminação de fitas seguido de usinagem ou por meio de processo de fundição centrífuga dependendo das dimensões e exigências de projeto.

Para o processo de fundição centrífuga, que é mais amplamente usado para mancais hidrodinâmicos de grandes dimensões, as ligas base de metal de coeficiente de atrito reduzido, que estarão em contato com o eixo, podem ser de bronze, alumínio ou liga de chumbo, em sua grande maioria, podendo existir outras. Neste processo, a carcaça de aço já está nas dimensões finais ou próximas da geometria desejada para o produto final. Esta estrutura é preparada (usinada, desengraxada, removida oxidação e aquecida) para a liga base fundida através de um processo de centrifugação. O processo de centrifugação consiste basicamente em vazar a liga fundida dentro da porção oca da carcaça de aço, enquanto esta gira em uma determinada rotação controlando a distribuição da liga no interior da peça e sendo posteriormente resfriada por jatos controlados de líquido refrigerante na porção externa de aço.

Para este último tipo de processamento existem diferentes variáveis que podem interferir na qualidade do produto final e que devem ser avaliadas com rigoroso critério para eliminação de divergências do processo, não conformidades e retrabalhos, ou até mesmo perda da peça. Na etapa de projeto, as características de resistência mecânica ou rigidez ao qual o mancal será submetido devem ser consideradas para determinar qual tipo de material, ou na sua grande maioria qual tipo de aço deverá ser utilizado (ARNOLD, 2005, p. 1-16). Já na etapa de fundição centrífuga propriamente dita, os parâmetros de controle de composição de liga base, temperatura de vazamento da liga base, temperatura do metal patente no momento do vazamento e condições de resfriamento, são as variáveis mais importantes a serem consideradas.

\section{Metodologia}

O procedimento de elaboração e implementação da metodologia de Análise dos Modos de Falhas e seus Efeitos na metalúrgica produtora de mancais, buchas e bronzinas, seguiu o ciclo PDCA (Planejar, Fazer, Verificar e Padronizar) para implementação. Inicialmente foi determinado o processo a ser estudado e no qual seria realizado a FMEA piloto e o escolhido foi o processo de produção de mancais especiais, devido ao maior histórico de não conformidades. Além disso, foi decidido pelo grupo focar nas etapas de fundição e processos antecessores a este, que também pelo histórico são os principais responsáveis pelo maior nível de problemas. Na sequência, formou-se a equipe multifuncional que participaria deste FMEA piloto e que daria continuidade nos projetos futuros. A equipe foi composta por profissionais dos setores de produção, qualidade, engenharia e 
planejamento e controle da produção.

Seguindo para o próximo passo, elaborou-se o fluxograma dos processos de produção de mancais especiais, peças da linha automotiva e produção de buchas e casquilhos de alumínio. Estes dois últimos processos, não participaram neste FMEA piloto, mas já foram elaborados para continuidade em projetos posteriores. Na etapa seguinte realizou-se a caracterização das funções de cada etapa do processo.

A identificação dos tipos, efeitos e causas das falhas procedeu-se com as análises em conjunto de cada integrante do grupo multifuncional e com as visões e conhecimentos dos operadores de produção de cada estação de trabalho envolvida. Nesta etapa foi utilizado o diagrama de causa e efeito como ferramenta para identificação de algumas falhas do processo. Ainda nestas análises, foram avaliados os índices de ocorrência, severidade, detecção e o grau de priorização de risco (GPR).

Foram acompanhados os indicadores de qualidade das peças produzidas e identificados os principais controles existentes do processo e pelo GPR foram projetadas as principais ações preventivas e corretivas para melhoria do processo. Deste modo, o plano de ação de melhorias foi elaborado utilizando-se o 5W2H como ferramenta guia.

As etapas seguintes do ciclo PDCA, executar, verificar e padronizar, ficaram de responsabilidade da empresa devido ao tempo para implementação das ações de melhoria e da necessidade de disponibilização de recursos.

\section{Resultados e discussão}

Iniciando o processo de implementação da FMEA na metalúrgica produtora de mancais, procedeu-se como primeira etapa a formação da equipe multifuncional para elaboração das etapas de execução e criação da FMEA. Por meio de históricos de defeitos, retrabalhos e refugos verificaram-se os principais tipos de defeitos existentes e a etapa do processo com maior índice de problemas. Como verificado no gráfico de Pareto dos índices de defeitos, figura 1, o maior problema encontrado no processo é o descolamento ou falta de aderência da liga base com o metal patente (metal base). Este defeito é mais recorrente no processo de produção de mancais especiais onde a variabilidade de tipos e tamanhos de peças é grande e não é uma produção seriada. 
Gráfico 1 - Não conformidades de processo

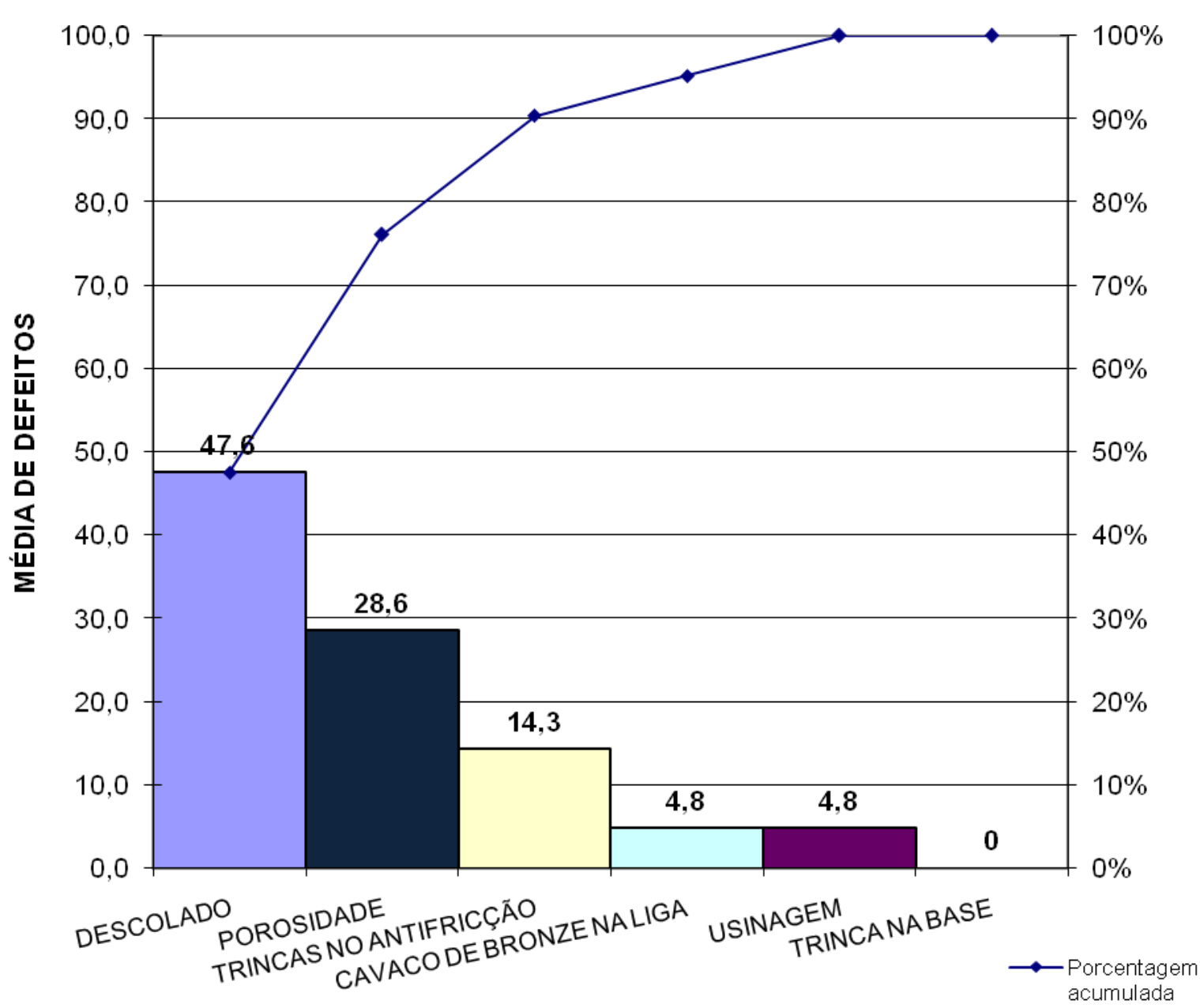

TIPOS DE DEFEITOS

Fonte: Pesquisa de campo (2014)

Com este levantamento a equipe optou por realizar a implementação da FMEA piloto no processo de produção de mancais especiais. Foi elaborado o fluxograma do processo, figura 2, para entendimento do passo a passo da produção e delineamento das áreas e possíveis não conformidades encontradas em cada seção do processo.

De todas as operações que podem afetar a fabricação, as etapas de preparação do metal base até resfriamento da liga de revestimento são as mais importantes e que tem influência direta no defeito de descolamento. Estas etapas envolvem controles de composição de material base, temperaturas, aspectos superficiais do metal patente e características próprias da operação de centrifugação.

Portanto, com este fluxograma torna-se mais evidente os principais pontos de controle que devem existir para manter um padrão de produção e onde atuar com respostas rápidas para correção de desvios de processo. 
Figura 1 - Fluxograma do processo de produção de mancais especiais

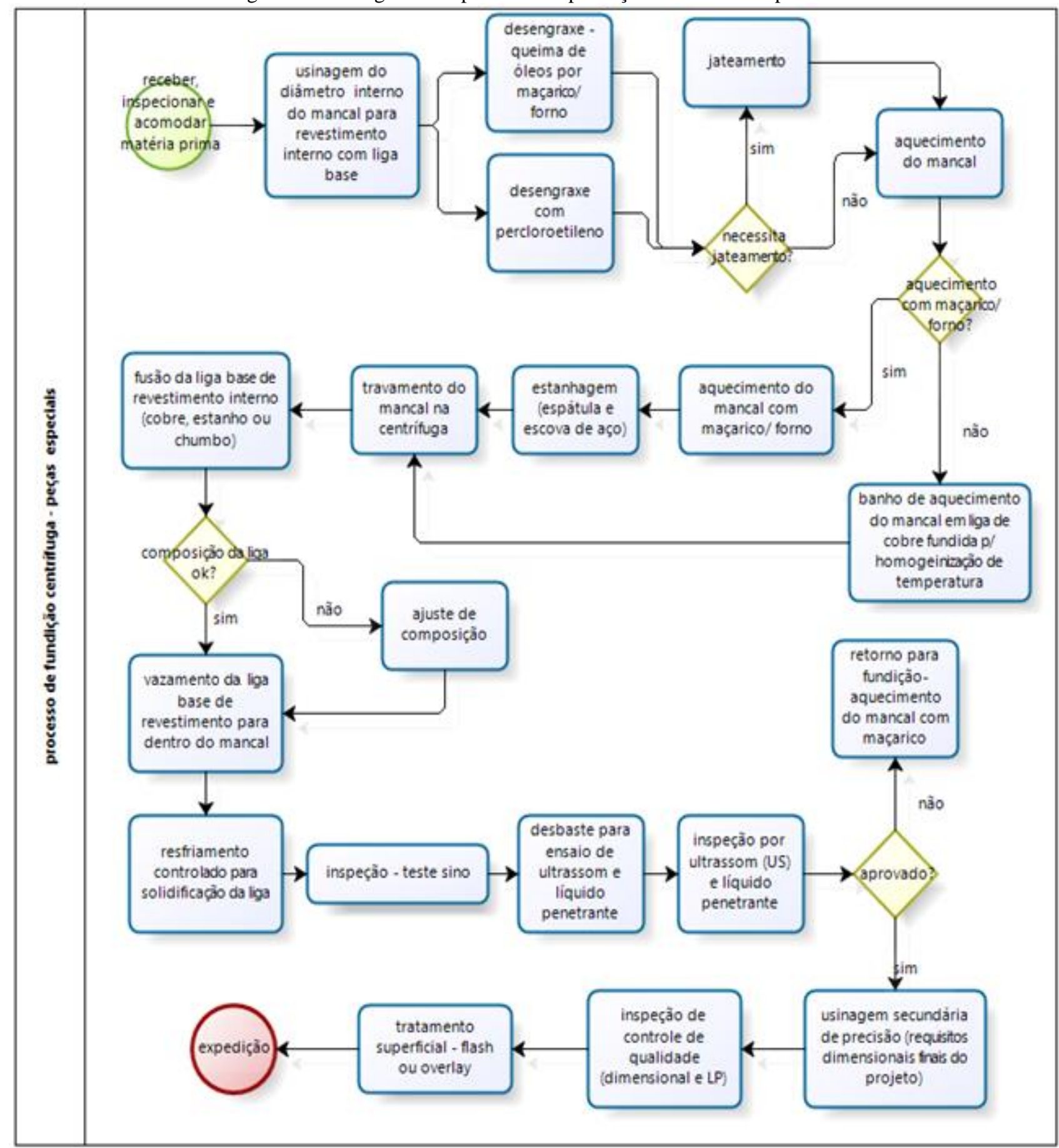

Fonte: Autoria própria (2014).

Após visualizar a sequência de operações do processo de produção, elaborou-se um diagrama de causa e efeito para entender quais possíveis causas poderiam gerar o principal defeito de fabricação que é o descolado ou falta de aderência. Este diagrama aborda os 6Ms de causas que são: mão de obra, máquina, método, medida, meio ambiente e material. Para cada um dos Ms foi realizado um brainstorming com a equipe para avaliar possíveis meios causadores do problema de descolamento. A figura 3 seguinte, apresenta alguns exemplos de causas relacionados ao efeito de falta de aderência, foram discutidos 26 possíveis causas nesta análise primária, numerados de 1 a 26 no diagrama. Uma das causas pontuadas e de grande importância é o método de vazamento e 
centrifugação que pode originar grandes gradientes de temperatura e interferir na aderência da liga no metal patente.

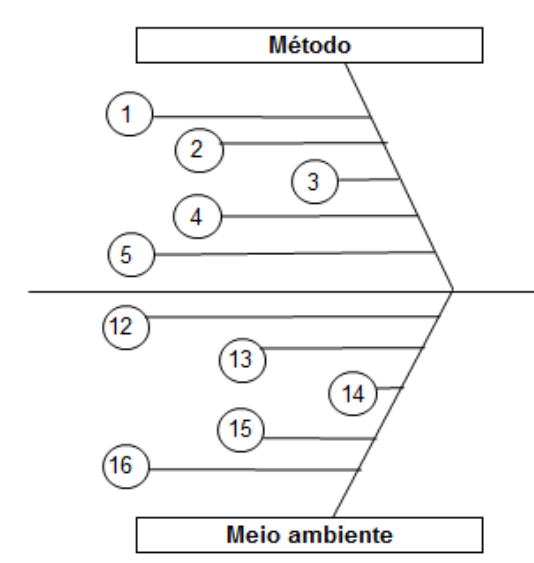

Figura 2 - Diagrama de causa e efeito

Fonte: Autoria própria (2014)

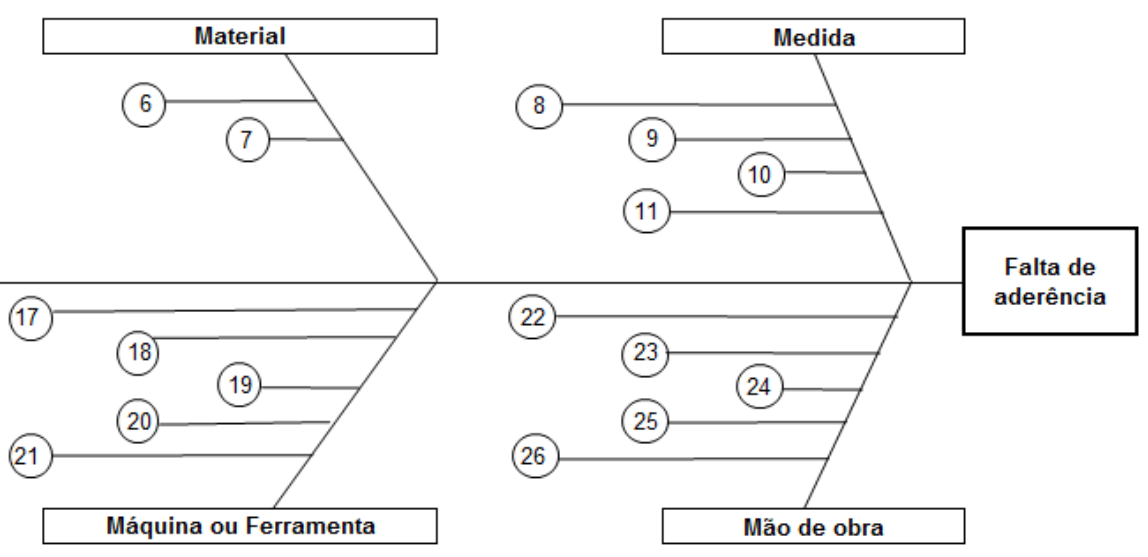

4-Método de vazamento e centrifugação permite ocorrer grandes gradientes de temperatura em diferentes partes da peça; 7-Equipamento de tamponamento das laterais dos mancais durante centrifugação com grande diferença de condutibilidade térmica e diferença de temperatura do metal patente; 9-Não existe controle da quantidade de temperatura reduzida entre aquecimento do mancal, travamento na centrífuga e vazamento da liga base; 15-Banhos químicos contaminados com partículas de óxidos e muita oleosidade de outros banhos (banhos saturados); 16-Layout dos fornos aumenta a dissipação de calor do mancal entre o período de travamento e vazamento da liga; 18Bicos de resfriamento com distribuição heterogênea do fluxo de água; 22-Remoção inadequada da oxidação e oleosidade da peça.

Seguindo o ciclo PDCA e a metodologia para elaboração da FMEA e tomando como base o fluxograma, caracterizou-se as funções do processo seguindo as etapas de produção, identificando os requisitos de cada função do processo e os possíveis modos de falha, seus efeitos e as causas prováveis destas falhas, toda esta metodologia utilizando o brainstorming para captar maior quantidade de modos de falhas possíveis. Com os indicadores de retrabalho, refugo e avaliação de engenharia de severidade de cada falha, quantificou-se os índices de severidade, ocorrência e detecção de cada falha, utilizando a tabela 1 de referência (ROTONDARO et al, 2002, p.159).

Estes valores resultaram no grau de priorização e risco (GPR), que identifica quais são os principais problemas do processo e que devem receber maior atenção para elaboração de um plano de contenção e melhorias mais imediato. A tabela 2 mostra algumas destas falhas com os valores de GPR mais altos. O efeito de falta de aderência foi novamente caracterizado tendo relação com uma possível causa de problema com o material de vedação do mancal, no processo de centrifugação que origina um gradiente de temperatura e é um provável responsável por alguns dos defeitos de descolamento. 
Tabela 2 - Exemplo da FMEA do processo de produção de mancais especiais

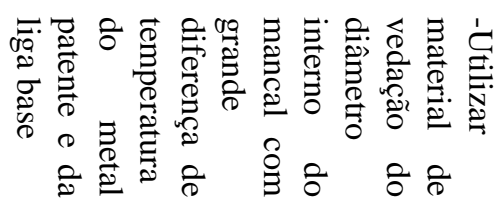

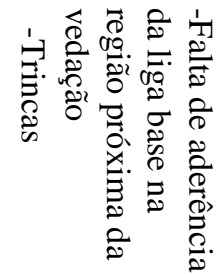

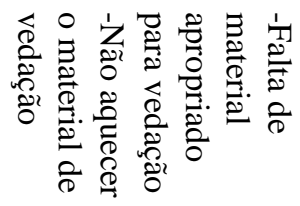

6

$\infty$

$\stackrel{8}{+}$

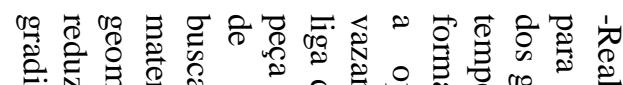

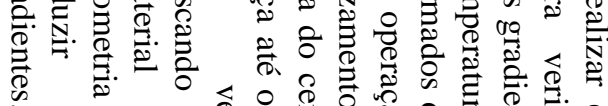

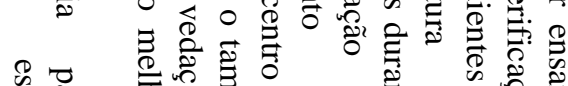

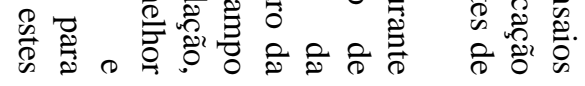
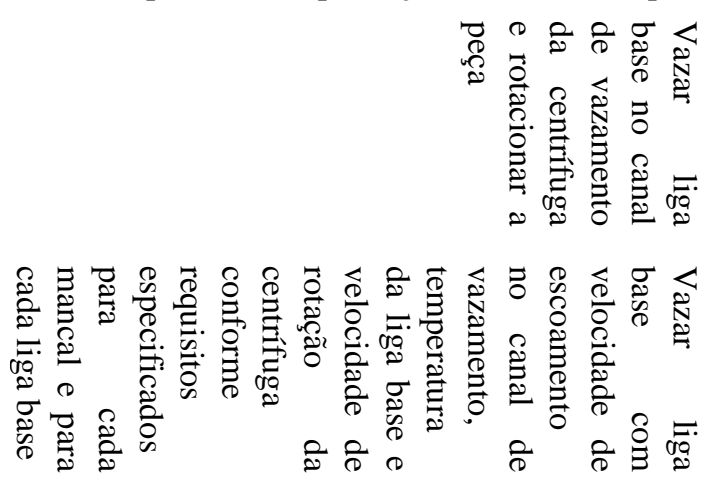

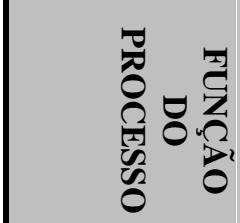

\section{$=0<\sigma<$}

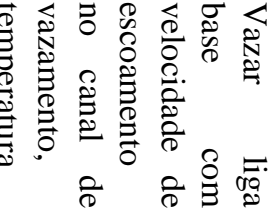

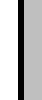
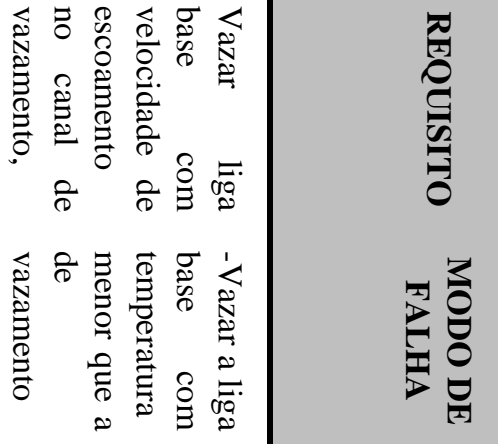

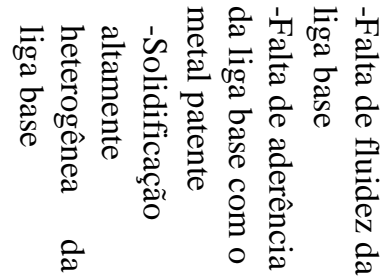

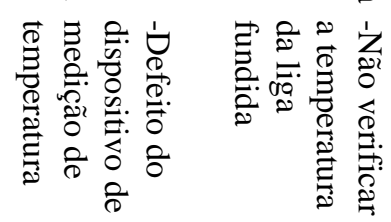
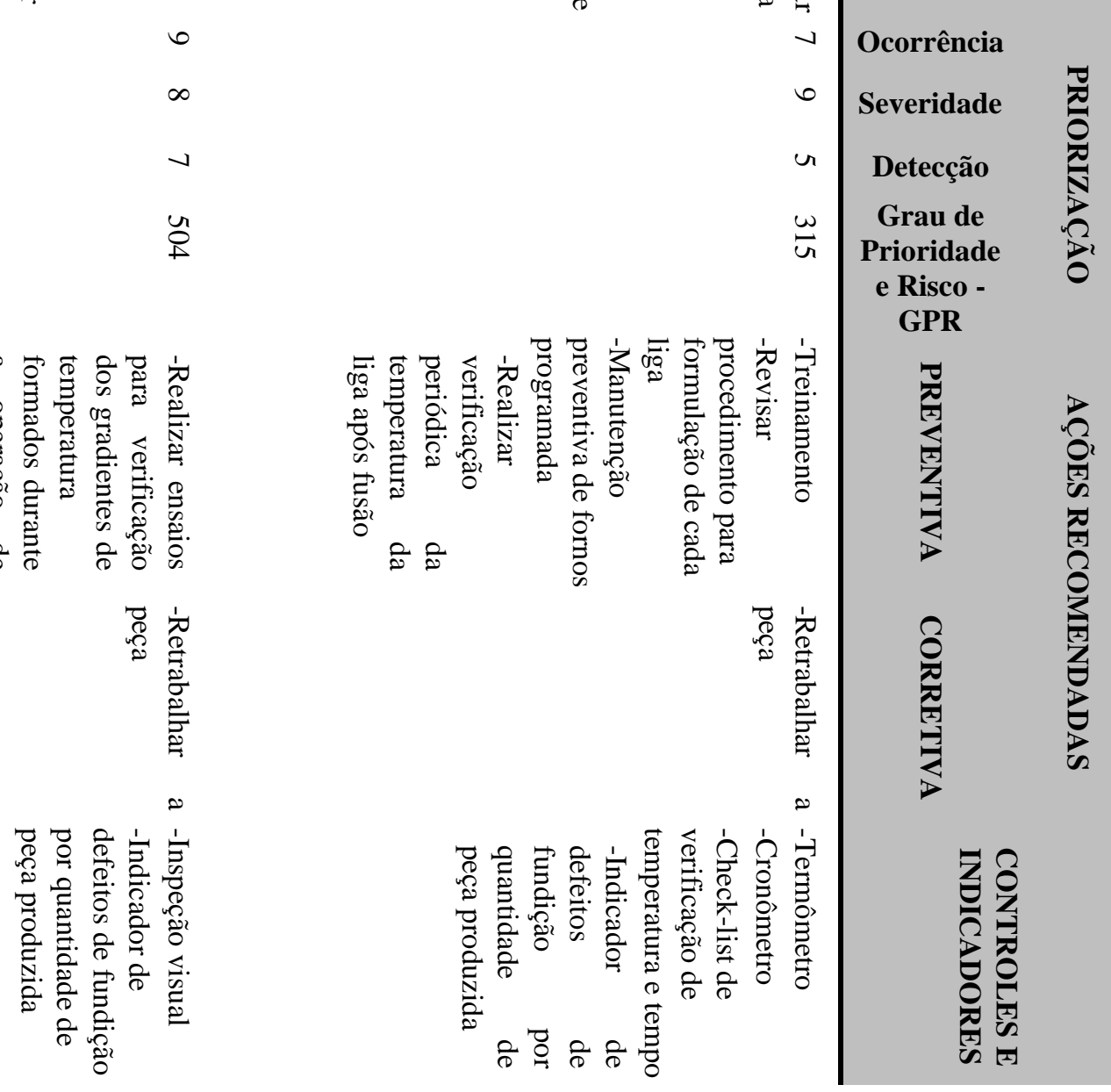

GPR
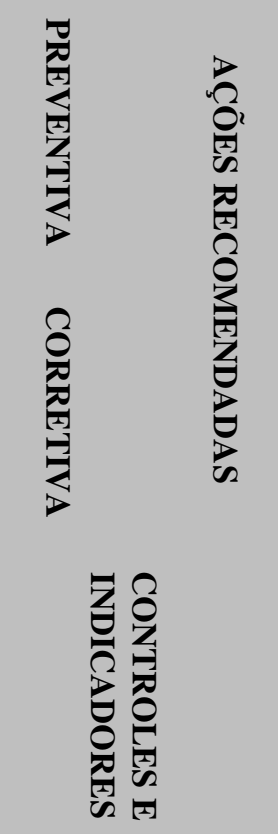

Fonte: Autoria própria (2014). 
Finalizando o passo inicial da FMEA e ainda na etapa do Plan do PDCA, elaboraram-se algumas ações preventivas e corretivas para melhoria do processo baseando-se no 5W2H como ferramenta de orientação para execução dos planos de ação. Para a falha de utilizar material de vedação do mancal no processo de centrifugação, a qual obteve um GPR de 540, o plano de ação preventivo é apresentado na tabela 3.

As ações de execução do plano de ação, verificação da eficácia e padronização dos resultados, seguindo o ciclo PDCA, ficaram de responsabilidade da equipe multifuncional, devido ao tempo de execução e a necessidade de liberação de recursos.

Tabela 3 - Exemplo de 5W2H como plano de ação para a falha de material de vedação na centrifugação

\begin{tabular}{|c|c|}
\hline Função & Resposta \\
\hline Quem? & Analista de Engenharia. \\
\hline O Quê? & $\begin{array}{l}\text { Realizar ensaios para avaliar o melhor material e dimensões para os principais mancais especiais } \\
\text { produzidos, avaliando as diferenças de gradientes de temperaturas formados durante processo de } \\
\text { fundição centrífuga e avaliar melhor temperatura de pré-aquecimento deste material de vedação. }\end{array}$ \\
\hline Quando? & Até 4 meses após início de implementação do FMEA. \\
\hline Onde? & Laboratórios de caracterização de materiais. \\
\hline Por quê? & $\begin{array}{l}\text { Para avaliar melhor material a ser usado que reduza efeitos de gradiente de temperatura e } \\
\text { consequentemente falha de descolamento da liga. }\end{array}$ \\
\hline Como? & $\begin{array}{l}\text { Utilizando softwares de simulação e realizando ensaios com algumas amostras para verificar os } \\
\text { resultados do simulador. }\end{array}$ \\
\hline $\begin{array}{l}\text { Quanto } \\
\text { Custa? }\end{array}$ & $\begin{array}{l}\text { Hora de trabalho do analista, compra de alguns corpos de prova, ensaio das amostras e hora de } \\
\text { simulação no software. }\end{array}$ \\
\hline
\end{tabular}
Fonte: Autoria própria (2014).

\section{Conclusão}

A utilização da metodologia da FMEA é uma excelente ferramenta para gestão de riscos e conhecer como é a real situação do processo e os defeitos que afetam a qualidade dos produtos. Este método permite identificar modos de falhas, seus efeitos e as causas envolvidas e se mostrou de grande valia para o processo de produção de mancais através de fundição centrífuga.

Um dos principais problemas da metalúrgica em estudo é o grande índice de retrabalho e até mesmo de refugo decorrente de grandes flutuações no processo e pela variabilidade de peças produzidas. Um dos defeitos mais presentes neste tipo de processo é a falta de aderência da liga base, registrado no histórico de defeitos, e que ainda não havia sido realizado um estudo para detalhar possíveis causas. A aplicação da FMEA neste processo e o mapeamento da sequência de produção, por meio de um fluxograma, mostraram-se bastante úteis para avaliar possíveis causas deste problema e elaborar um plano de ação para reduzi-lo e, como meta, eliminar o mesmo do processo de produção de mancais especiais.

Este tipo de metodologia é requisito obrigatório para a contratação de fornecedores dos mais diversos segmentos. A exigência dos clientes por produtos e processos livres de defeitos faz aumentar a utilização da FMEA principalmente em empresas, nas quais, um simples defeito pode 
ocasionar um risco à vida do usuário. Em empresas do ramo automobilístico, aeronáutico e hospitalar, a aplicação da FMEA já faz parte da rotina da organização. Esta ferramenta pode ser aplicada em qualquer setor e em empresas de qualquer porte, pois não exige grande investimento na sua elaboração, apenas um conhecimento mais aprofundado do fluxo das operações ou de detalhes dos requisitos de um produto ou serviço.

\begin{abstract}
The increasing competitiveness in all sectors of the economy and the need to improve production while avoiding waste, increasing quality and producing an attractive price has gained ground in discussions of companies from all segments, to seek techniques and methodologies that help achieve these goals. One of the methods widely used for this risk management is the FMEA (Failure Mode and Effect Analysis), which aims to identify possible failure of a project, process, product or service points. This work was prepared as a result of a problem that affects many industries, including metallurgy industries: rework the production process. The rework involves the repetition of an activity caused by failure problems of manpower, material, design or process problems. In the manufacturing process of bearings, there is a wide range of variables that can influence the final result and generate a significant impact on product quality. To suppress this impact and gain a competitive advantage in the market, FMEA was selected as a method to reduce process failures. The aim of this paper is to develop a plan according to the FMEA methodology and propose action plans for the identified problems in the production process. Also aims to demonstrate the effectiveness of the methodology in spreading a culture focused on continuous improvement.
\end{abstract}

Key-words: analyze; failure; risk; bearings; flowchart.

\title{
Referências Bibliográficas
}

ASSOCIAÇÃO BRASILEIRA DE NORMAS TÉCNICAS. ISO/TS 16949: Sistema de Gestão da Qualidade Requisitos Particulares para aplicação da ABNT NBR ISO 9001:2000 para organizações de produção automotiva e peças de reposição pertinentes. Rio de Janeiro: ABNT, 2004.

ASSOCIAÇÃO BRASILEIRA DE NORMAS TÉCNICAS. NBR ISO 31000: Gestão de Riscos - Princípios e Diretrizes. Associação Brasileira de Normas Técnicas, Rio de Janeiro: ABNT, 2009.

ARABIAN-HOSEYNABADI, H.; ORAEE, H.; TAVNER, P.J. Failure Modes and Effects Analysis (FMEA) for wind turbines. International Journal of Electrical Power and Energy Systems, v. 32, 2010.

ARNOLD, K. F.. Validação experimental de uma metodologia de cálculo da vida à fadiga para mancais hidrodinâmicos. 2005, 140 f. Dissertação (Mestrado Profissional em Engenharia Automotiva) - Escola Politécnica da Universidade de São Paulo, São Paulo, 2005.

Automotive Industry Action Group (AIAG). Análise de modo e efeitos de falha potencial (FMEA) - Manual de Referência. Tradução Instituto de Qualidade Automotiva (IQA). 4 ed. São Paulo: IQA, 2008. 141 p.

BLAUTH, R.; BLAUTH, R.. Gestão da Qualidade. 2 ed. Curitiba: IESDE Brasil S.A., 2009.

BRASILIANO, A. C. R. Gestão e análise de riscos corporativos: Método Brasiliano avançado. São Paulo: Sicurezza, 2009.

HARGREAVES, L.; et al. Qualidade em prestação de serviços. 2 ed. Rio de Janeiro: Senac Nacional, 2001. p.12.

HELMAN, H.; ANDERY, P. R. P. Análise de falhas (Aplicação dos métodos de FMEA e FTA) - Ferramentas da Qualidade. v. 11, Belo Horizonte, MG: Fundação Christiano Ottoni, Escola de Engenharia da UFMG, 1995. 
MARSHALL JUNIOR, I. et al. Gestão da qualidade. 10 ed. Rio de Janeiro: FGV, 2010.

ROTONDARO, R.G. SFMEA: Análise do modo e efeito de falha em serviços - aplicando técnicas de melhorias em serviços. Produção, v. 12, n. 2, p. 54-62, 2002. cross ref

ROTONDARO, R. G. et al. Seis Sigma: estratégia gerencial para a melhoria de processos, produtos e serviços. São Paulo: Atlas, 2002.

TOLEDO, J. C.; et al. Qualidade: gestão e métodos. Rio de Janeiro: LTC, 2013.

VASCONCELloS JR, J. P. C. de; MIGUEL, P. A. C. Implementação de FMEA em uma empresa de máquinasferramenta. In: ENCONTRO NACIONAL DE ENGENHARIA DE PRODUÇÃO, 19; 1999, Rio de Janeiro, RJ. Anais...Rio de Janeiro, 1999.

VINODH, S.; SANTHOSH, D. Application of FMEA to an automotive leaf spring manufacturing organization. The TQM Journal, v. 24, n. 3, p. 260-274, 2012. crossref

WERKEMA, M. C. C. Criando a Cultura Seis Sigma. Rio de Janeiro: Editora Qualitymark, 2002.

WERKEMA, M. C. C. Ferramentas Estatísticas Básicas para o Gerenciamento de Processos. Belo Horizonte: Fundação Christiano Ottoni, 1995.

\section{Dados dos autores}

Nome completo: André Victor Traleski

Filiação institucional: Faculdade União

Função ou cargo ocupado: Aluno pós-graduado

Endereço completo para correspondência: Rua Baltazar Lisboa, 455, Bairro: Ronda, Ponta Grossa-

PR, Brasil. CEP: 84051-090.

Telefones para contato: (42) 3224-2005

e-mail:andre_traleski@hotmail.com

\section{Nome completo: Henry Stepien}

Filiação institucional: Faculdade União

Função ou cargo ocupado: Aluno pós-graduado

Endereço completo para correspondência: Rua Londrina, 323, Bairro: Santo Antônio, Ponta GrossaPR, Brasil. CEP: 84053-320.

Telefones para contato: (42) 3223-3498/ (42) 9926-1233

e-mail:henryst@bol.com.br

\section{Nome completo: Ricardo Augusto Blauth}

Filiação institucional: Universidade Positivo

Função ou cargo ocupado: Professor Assistente

Endereço completo para correspondência: Av. Cândido Hartmann, 3045 casa J. Curitiba-PR, Brasil. CEP 82010-000. 
Telefones para contato: (41) 8428-4444

e-mail: ricardo@eqpconsultoria.com.br

Submetido em: 2014-10-21

Aceito em: 2015-05-07 